\title{
Electronic structure of CdSe-ZnS 2D nanoplatelets
}

Hervé Cruguel, Clément Livache, Bertille Martinez, Silvia Pedetti, Debora Pierucci, Eva Izquierdo, Marion Dufour, Sandrine Ithurria, Hervé Aubin, Abdelkarim Ouerghi, Emmanuelle Lacaze, Mathieu G. Silly, Benoit Dubertret, and Emmanuel Lhuillier

Citation: Appl. Phys. Lett. 110, 152103 (2017); doi: 10.1063/1.4980065

View online: http://dx.doi.org/10.1063/1.4980065

View Table of Contents: http://aip.scitation.org/toc/apl/110/15

Published by the American Institute of Physics

\section{Articles you may be interested in}

Independent tuning of excitonic emission energy and decay time in single semiconductor quantum dots Appl. Phys. Lett. 110, 151102151102 (2017); 10.1063/1.4979481

van der Waals epitaxy of CdS thin films on single-crystalline graphene

Appl. Phys. Lett. 110, 153104153104 (2017); 10.1063/1.4980088

Orientation-specific transgranular fracture behavior of CVD-grown monolayer MoS2 single crystal Appl. Phys. Lett. 110, 153105153105 (2017); 10.1063/1.4979974

Three-terminal quantum-dot thermal management devices

Appl. Phys. Lett. 110, 153501153501 (2017); 10.1063/1.4979977

Quantum interference of two photons emitted from a luminescence center in GaAs: N Appl. Phys. Lett. 110, 152102152102 (2017); 10.1063/1.4979520

Fabrication of high-quality GaAs-based photodetector arrays on $\mathrm{Si}$ Appl. Phys. Lett. 110, 153505153505 (2017); 10.1063/1.4980122






\title{
Electronic structure of CdSe-ZnS 2D nanoplatelets
}

 \\ Debora Pierucci, ${ }^{3}$ Eva Izquierdo, ${ }^{2}$ Marion Dufour, ${ }^{2}$ Sandrine Ithurria, ${ }^{2}$ Hervé Aubin, ${ }^{2}$ \\ Abdelkarim Ouerghi, ${ }^{4}$ Emmanuelle Lacaze, ${ }^{1}$ Mathieu G. Silly, ${ }^{5}$ Benoit Dubertret, ${ }^{2}$ \\ and Emmanuel Lhuillier ${ }^{1, b)}$ \\ ${ }^{1}$ Sorbonne Universités, UPMC University Paris 06, CNRS-UMR 7588, Institut des NanoSciences de Paris, \\ 4 place Jussieu, 75005 Paris, France \\ ${ }^{2}$ Laboratoire de Physique et d'Etude des Matériaux, ESPCI-ParisTech, PSL Research University, \\ Sorbonne Universités UPMC University Paris 06, CNRS, 10 rue Vauquelin, 75005 Paris, France \\ ${ }^{3}$ Univ. Grenoble Alpes, CNRS, Institut Néel, F-38000 Grenoble, France \\ ${ }^{4}$ Centre de Nanosciences et de Nanotechnologies, CNRS, University of Paris-Sud, Université Paris-Saclay, \\ C2N, Marcoussis 91460, France \\ ${ }^{5}$ Synchrotron-SOLEIL, Saint-Aubin, BP48, F91192 Gif sur Yvette Cedex, France
}

(Received 10 February 2017; accepted 31 March 2017; published online 11 April 2017)

\begin{abstract}
Among colloidal nanocrystals, 2D nanoplatelets (NPLs) made of cadmium chalcogenides have led to especially well controlled optical features. However, the growth of core shell heterostructures has so far been mostly focused on CdS shells, while more confined materials will be more promising to decouple the emitting quantum states of the core from their external environment. Using $\mathrm{k} \cdot \mathrm{p}$ simulation, we demonstrate that a $\mathrm{ZnS}$ shell reduces by a factor 10 the leakage of the wavefunction into the surrounding medium. Using X-ray photoemission (XPS), we confirm that the $\mathrm{CdSe}$ active layer is indeed unoxidized. Finally, we build an effective electronic spectrum for these $\mathrm{CdSe} / \mathrm{ZnS}$ NPLs on an absolute energy scale which is a critical set of parameters for the future integration of this material into optoelectronic devices. We determine the work function (WF) to be $4.47 \mathrm{eV}$ while the material is behaving as an $n$-type semiconductor. Published by AIP Publishing.

[http://dx.doi.org/10.1063/1.4980065]
\end{abstract}

Colloidal nanocrystals are attracting more research and industrial efforts because of their tunable optical features from UV to THz. ${ }^{1}$ Among them, colloidal quantum wells, ${ }^{2}$ also called nanoplatelets ${ }^{3,4}$ (NPL), are a special class of colloidal nanocrystals, which offers an improved control on the absorption and emission properties. ${ }^{5}$ NPLs are strongly anisotropic objects with a large lateral extension (i.e., above the Bohr radius) and a perfectly controlled thickness. Because of their atomic scale control of the only confined direction, ${ }^{6}$ NPLs present a narrow photoluminescence (PL) linewidth. The PL can be as narrow as $10 \mathrm{~nm}$ for an emission in the green $(510 \mathrm{~nm})$, which is two to three times narrower than the value conventionally obtained for spherical nanoparticles whose properties are limited by the size distribution. Such narrow luminescence is interesting for the design of light emitting diodes (LEDs) ${ }^{7,8}$ and displays with enhanced gamut. In addition, the $2 \mathrm{D}$ dielectric screening ${ }^{9,10}$ leads to a large binding energy (BE), which prompts the luminescence lifetime and has led to lasing properties ${ }^{11}$ with reduced threshold. ${ }^{12,13}$ Soon after their first synthesis, the possibility to revisit the growth of heterostructures with $2 \mathrm{D}$ objects has emerged. It thus became possible to grow core-shell ${ }^{14,15}$ and core-crown ${ }^{16}$ heterostructures with type I and type $\mathrm{II}^{17}$ band alignments.

With the aim of obtaining a strong luminescence, the current efforts have been focused on CdS and CdZnS shells with a low $\mathrm{Zn}$ content $(<30 \%)$. CdS offers the possibility to grow a larger shell because of the vicinity of the $\mathrm{CdS}$ and

\footnotetext{
${ }^{a}$ H. Cruguel, C. Livache, and B. Martinez contributed equally to this work.

b) Author to whom correspondence should be addressed. Electronic mail: el@insp.upmc.fr
}

CdSe lattice constant and leads to a reduced blinking. ${ }^{18}$ However, in $\mathrm{CdSe} / \mathrm{CdS}$ heterostructures, the electron gets delocalized all over the two materials because of the limited conduction band-offset of CdSe and CdS. As a result, the protection toward the environment remains limited. To obtain a stronger exciton confinement, materials with a wider band offset are necessary. Here, we investigate the electronic properties of $\mathrm{CdSe} / \mathrm{ZnS}$ nanoplatelets using X-ray photoemission and numerical simulations. In particular, we aim to determine the effective electronic levels of these NPLs on an absolute energy scale. This step is crucial for the design of future devices such as LED based on the CdSe/ZnS NPL, in particular, for the fabrication of ohmic contacts injecting efficiently holes and electrons.

To motivate the growth of $\mathrm{CdSe} / \mathrm{ZnS}$ NPLs, we first investigate their electronic structure using numerical simulations based on a k.p method. For such nanocrystals, it has often been argued that the tight binding approach leads to more accurate determination of the electronic spectrum; ${ }^{8}$ however, tight binding methods are difficult to apply to heterostructures, while their treatment by $\mathrm{k} \cdot \mathrm{p}$ simulation is quite straightforward. Since the lateral extension of the NPL is larger than their material Bohr radius, we conduct 1D simulation along the NPL thickness. We solve the time independent Schrodinger equation $\left[-\frac{\hbar^{2}}{2} \frac{d}{d z} \frac{1}{m^{*}(z)} \frac{d}{d z}+V(z)\right] \xi(z)=E \xi(z)$ thanks to a shooting method $^{19}$ (see Section I of the supplementary material for details) for the heavy hole and conduction band and determine the electronic spectrum and the associated wavefunctions $\xi$. Here, $\hbar$ is the reduced Planck constant, $m^{*}$ the effective mass, $V$ the band profile, and $E$ the eigen energy of the system. The parameters given in Table I have been used as input material 
TABLE I. Band structure parameters used to solve the Schrödinger equation.

\begin{tabular}{lccc}
\hline \hline Material & CdSe & CdS & ZnS \\
\hline Band gap $(\mathrm{eV})$ & 1.66 (Ref. 20) & 2.4 (Ref. 17) & 3.7 (Ref. 17) \\
$\mathrm{m}_{\mathrm{e}}\left(\mathrm{m}_{0}\right)$ & 0.11 (Ref. 21) & 0.14 (Ref. 22) & 0.37 (Ref. 23) \\
$\mathrm{m}_{\mathrm{h}}\left(\mathrm{m}_{0}\right)$ & 0.44 (Ref. 21) & 0.51 (Ref. 19) & 0.57 (Ref. 20) \\
Valence band offset & 0 & -0.42 (Ref. 24) & -0.6 (Ref. 21) \\
with CdSe $(\mathrm{eV})$ & & & \\
\hline \hline
\end{tabular}

parameters. We apply this model to the case of an alloy barrier with $\mathrm{Cd}_{1-\mathrm{x}} \mathrm{Zn}_{\mathrm{x}} \mathrm{S}$ content. In this case, the band parameters are taken as a linear extrapolation of the $\mathrm{CdS}$ and $\mathrm{ZnS}$ parameters, which neglects any possible bowing effects.

Figure 1(a) presents the typical energy profile of the CdSe/ZnS NPLs and the first two wavefunctions for electrons and holes. In Figure 1(b), we present the ground state wavefunction for the conduction band as we tune the $\mathrm{Zn}$ content in the barrier. As the zinc content increases, the electron wavefunction is more and more localized in the CdSe core. Thanks to this simulation, we can now quantify how much of the wavefunction is actually experiencing the surface. To do so, we plot the magnitude of the wavefunction at the interface between the shell and the surrounding medium (Figure $1(\mathrm{c}))$. For both electrons and holes, we observe a decrease in the magnitude of the wavefunction at the surface. The decay is particularly striking for the electron with a decrease by a factor 10 . This electron/hole asymmetry results from the fact that the hole is already confined whatever is the $\mathrm{Zn}$ content, while the electron is partly delocalized in the shell at a low $\mathrm{Zn}$ content. As a result, with CdSe/ZnS NPLs, we can expect a lower sensitivity to the environment and a higher stability of the performances.

The CdSe/ZnS NPLs have been provided by Nexdot. They come under the form of parallelepipeds with a lateral extension of $15 \mathrm{~nm} \times 40 \mathrm{~nm}$ and a thickness of $\approx 5 \mathrm{~nm}$ (Figure 2(b)). Their absorption presents two main features attributed to the heavy hole to conduction band transition and the light hole to conduction band transition (see Figure 2(a)). The lowest energy peak is at $600 \mathrm{~nm}$. The photoluminescence signal is peaked at $615 \mathrm{~nm}(2 \mathrm{eV})$ and presents a full width at half


FIG. 2. (a) Absorption and luminescnce spectra of the CdSe/ZnS NPL. (b) TEM image of the CdSe/ZnS NPL.

maximum (FWHM) of $29 \mathrm{~nm}$. This absorption is quite shifted compared to the core only structure, which presents a PL signal at $510 \mathrm{~nm}$. This means that the wavefunction is partly delocalized within the $\mathrm{ZnS}$, as expected from Figure 1. However, this delocalization is weaker than that for CdS shell for which the PL signal is observed above $635 \mathrm{~nm},{ }^{11}$ as expected from our simulation. Since these materials are designed typically to be used as phosphor for displays and LED, their integration into a matrix is critical to prevent the air oxidation of the particle and provide thermal extraction of the generated heat. In the supplementary material, we compare the behavior of the CdSe/ZnS NPL with the CdSe/CdS NPL obtained through the conventional way of the literature. ${ }^{13,14} \mathrm{We}$ observe a significant drop of PL efficiency for the CdS shell, while the Zn containing material remains very bright. In addition, the PL efficiency keeps decreasing as a function of time with the $\mathrm{CdS}$ shell, while for the $\mathrm{ZnS}$ shell, the PL intensity is almost doubled (Figure S4 in the supplementary material). To better understand this material with enhanced stability, we now investigate experimentally its electronic structure.

X-ray photoemission (XPS) experiments were carried out at Synchrotron SOLEIL on the TEMPO beamline. ${ }^{25}$ During the XPS measurements, the photoelectrons were detected at normal emission and at $46^{\circ}$ from the polarization vector $\vec{E}$. We first prepare a conductive substrate by depositing $80 \mathrm{~nm}$ of gold on a Si wafer. The NPLs are then dropcasted on the


FIG. 1. (a) Conduction and valence band profiles of the CdSe/ZnS NPL along the confined direction. The two first wavefunctions of the valence and conduction bands have also been displayed. Each of them has been shifted from its Eigen energy. (b) Wavefunctions associated with the conduction band fundamental state for different $\mathrm{Zn}$ contents. (c) Magnitude of the electron and hole wavefunctions at the interface with a surrounding medium as a function of the $\mathrm{Zn}$ content in the shell. 
substrate. Due to the inelastic mean free path of photoelectrons, most of the XPS signals will come from the alkane chain ( $2 \mathrm{~nm}$ long) of the ligand. We consequently chose to make a ligand exchange toward ethanedithiol (EDT) capping $(0.5 \mathrm{~nm}$ long) to maximize the contribution coming from the NPL. To do so, the film is dipped in a $1 \%$ mass solution of EDT in ethanol and then rinsed with fresh ethanol. Several deposition steps are repeated to build a $100 \mathrm{~nm}$ thick film. We also take care to let a part of the gold substrate uncoated to use it as an energy reference. In particular, the binding energies (BE) were calibrated using the $\mathrm{Au} 4 \mathrm{f}_{7 / 2}$ peak $\left(\mathrm{Au} 4 \mathrm{f}_{7 / 2}\right.$ at $\mathrm{BE}=84 \mathrm{eV}$ ). The accuracy of the BE calibration was estimated to be around $0.1 \mathrm{eV}$. One should note that the NPL film remains luminescent in spite of this ligand exchange. The spectra were acquired with a photon energy of $600 \mathrm{eV}$ in order to be surface sensitive. ${ }^{26}$ The signal overview is presented in Figure 3(a). In addition to the Au substrate contribution, we observe contribution of $\mathrm{C}$ and $\mathrm{O}$ coming from the ligands, as well as peaks from $\mathrm{Cd}, \mathrm{Se}, \mathrm{Zn}$, and $\mathrm{S}$.

In $\mathrm{CdSe} / \mathrm{ZnS}$, the wavefunctions are mostly localized within CdSe. It is of utmost importance that the emissive material is indeed the one expected and that no additional contribution such as oxide gets involved in the electronic states. We hence focus on the CdSe contribution. The Cd 3d peak can be fitted by a doublet with a $6.7 \mathrm{eV}$ spin orbit splitting (Figure 3(b)). For the fit, we use a pseudo-Voight curve with a Gaussian weight of $80 \%$. Only one contribution is observed, and the $\mathrm{Cd} 3 \mathrm{~d}_{5 / 2}$ peak appears at $404.91 \mathrm{eV}$ with a FWHM of $0.89 \mathrm{eV}$. In fact, we do not find evidence for the presence of $\mathrm{CdO}$, whose $\mathrm{Cd} 3 \mathrm{~d}_{5 / 2}$ binding energy is $404.2 \mathrm{eV}$ (a rare case in which the binding energy of the oxide is smaller than that of the metallic state, namely, $404.9 \mathrm{eV}){ }^{27}$ The Se $3 \mathrm{~d}$ peak is also coming under the form of a single contribution, which is a doublet with a $0.86 \mathrm{eV}$ spin orbit splitting. The Se $3 \mathrm{~d}_{5 / 2}$ peak presents a binding energy of $53.77 \mathrm{eV}$ for a $0.72 \mathrm{eV}$ FWHM. The presence of Se oxide ${ }^{28}$ $\left(\mathrm{SeO}_{2}\right)$ is expected to come under the form of a broad peak in the $57-60 \mathrm{eV}$ range and is not observed here. We can thus conclude that the CdSe of the $\mathrm{CdSe} / \mathrm{ZnS}$ heterostructure is not oxidized in spite of the air processing of the NPL and in spite of the ligand exchange. In this sense, the $\mathrm{ZnS}$ plays its protective role.

Finally, we use XPS to determine the work function and absolute energy of the valence band in order to determine the absolute energy (i.e., $v s$ vacuum) spectrum of the CdSe/ $\mathrm{ZnS}$ heterostructure. The work function (WF) of the sample was determined via the measurement of the secondary electron (SE) edge. In order to ensure that the SE has a kinetic energy (KE) higher than the analyzer vacuum level, the sample is negatively biased $(-9 \mathrm{~V})$ with respect to the analyzer. The position of the secondary electron cut-off ( $\left.\mathrm{KE}_{\text {cutoff }}\right)$ is measured by extrapolating the edge of the peak to the zero


FIG. 3. (a) Overview of the X-ray photoemission signal for a thin film of the CdSe/ZnS NPL deposited on a gold substrate. The incident photon energy is equal to $600 \mathrm{eV}$. (b) Zoom of the $\mathrm{X}$-ray photoemission spectrum of the area of the Cd 3d peak. (c) Zoom of the X-ray photoemission spectrum of the area of the Se $3 \mathrm{~d}$ peak. 

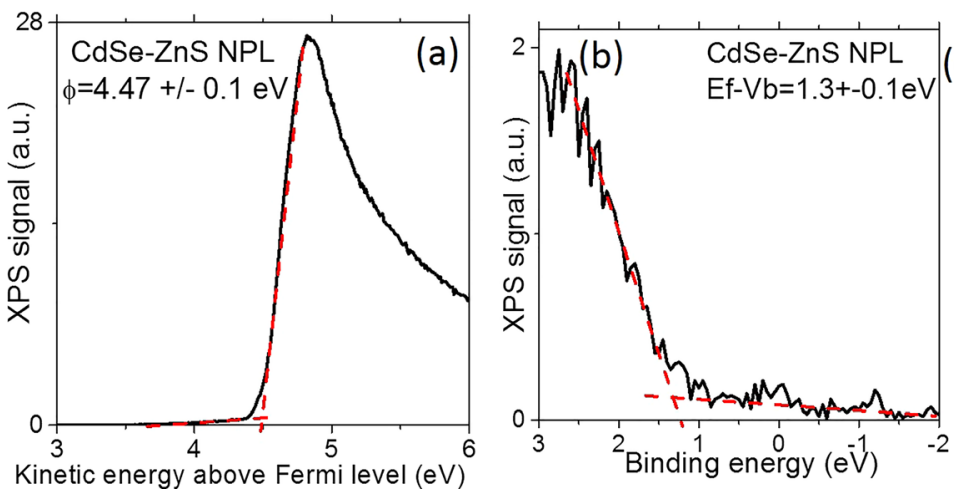

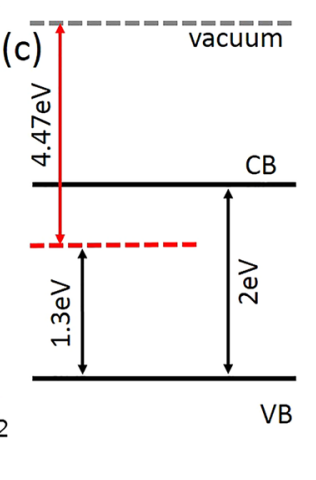

FIG. 4. (a) Cut off of the secondary electron for a thin film of the CdSe/ ZnS NPL deposited on a gold substrate and used to determine the work function of the film. (b) Top of the valence band photoemission signal for the same film. (c) Reconstructed electronic structure for the CdSe/ZnS NPL. baseline (Figure 4(a)). The photon energy $h v$ is calculated precisely by measuring the $1^{\text {st }}$ and $2^{\text {nd }}$ order spectra of the $\mathrm{Au} 4 \mathrm{f}$ core level. The kinetic energy of the $\mathrm{Au} 4 \mathrm{f}_{7 / 2}$ $\left(\mathrm{KE}_{\mathrm{Au} 4 \mathrm{f} 7 / 2}\right.$ (polarized)) and its known binding energy $(84 \mathrm{eV}$ according to the literature) $\mathrm{BE} \mathrm{Au} 4 \mathrm{f}_{7 / 2}$ are used as references. Then, the work function of the sample is given by $\mathrm{WF}=\mathrm{hv}+\mathrm{Ek}$ (cut off) $-\left(\mathrm{KE}_{\mathrm{Au} 4 \mathrm{f} 7 / 2}(\right.$ polarized $\left.)+\mathrm{BE}_{\mathrm{Au} 4 \mathrm{f} 7 / 2}\right)$. We found a value of $4.47 \mathrm{eV} \pm 0.1 \mathrm{eV}$ for the work function of the $\mathrm{CdSe} / \mathrm{ZnS}$ NPL sample.

The valence band energy with respect to the Fermi energy is determined by fitting the low binding energy part of the spectrum. We determine that the valence is $1.3 \mathrm{eV} \pm 0.1 \mathrm{eV}$ below the Fermi level. Given the fact that the $\mathrm{CdSe} / \mathrm{ZnS}$ presents a $2 \mathrm{eV}(615 \mathrm{~nm})$ band gap according to their photoluminescence signal (Figure 2(a)), we conclude that the material is slightly $n$-type with a Fermi level located at $\approx 2 / 3$ of the band gap. This $n$-doping is consistent with previous transport $^{29}$ and optical ${ }^{30}$ measurements conducted on cadmium chalcogenide nanocrystal materials.

Thanks to the obtained band structure, we would like to propose possible electrodes for the design of electron and hole injecting contact. Given the energy of the valence band (5.77 eV vs vacuum) and of the conduction band (3.77 eV vs vacuum), electron and hole injection can be efficiently achieved using respectively arsenic $(\mathrm{WF}(\mathrm{As})=3.75 \mathrm{eV})$ and platinum electrodes $(\mathrm{WF}(\mathrm{Pt})=5.65 \mathrm{eV})$. However, such couple presents two main drawbacks which are the toxicity and the lack of transparency. If transparent electrodes are necessary, we rather suggest the use a $\mathrm{MoO}_{3}\left(\mathrm{WF}\left(\mathrm{MoO}_{3}\right)>6 \mathrm{eV}\right)$ or a $\mathrm{NiO}(\mathrm{WF}(\mathrm{NiO})=5.4 \mathrm{eV})$ layer coupled with an ITO transparent electrode as a hole injector. For the electron injection, the [6,6]-phenyl-C61-butyric acid methyl ester (PCBM), a fullerene derivative, can be used as an electron transport layer because of its conduction band energy of $3.8 \mathrm{eV}^{31}$

The growth of well confined 2D heterostructured NPL is critical to achieve high PL efficiency and well defined cut off wavelength with materials poorly sensitive to their environment. Our numerical simulation shows that a $\mathrm{ZnS}$ shell reduces by a factor 10 the magnitude of the wavefunction at the interface compared to $\mathrm{CdS}$, which makes the $\mathrm{ZnS}$ shell promising to design materials with enhanced stability. It is critical to probe the electronic structure of these new colloidal materials, which has been done using X-ray photoemission. We first confirmed that the optically active layer of the heterostructure comes under a non-oxidized form. In addition, we determine the work function of the material to be
$4.47 \mathrm{eV}$, while the Fermi level is located at $2 / 3$ of the band gap, suggesting an $n$-type nature of this semiconductor. This work will pave the way for the integration of the $\mathrm{CdSe} / \mathrm{ZnS}$ NPL into optoelectronic devices with well-designed ohmic contact.

See supplementary material for discussion about the numerical resolution of the Schrödinger equation and the comparison of the optical properties of CdSe/CdS NPLs with CdSe/ZnS NPLs.

We thank Nicolas Goubet for his help with the PL setup. S.I. and B.D. acknowledge the support from Agence Nationale de la Recherche through grant Nanodose and H2DH, respectively. E.L. and S.I. are also supported by the Region Ile-de-France in the framework of DIM Nano-K, from ANR within the Investissements d'Avenir programme ANR-11-IDEX-0004-02, and within the framework of the Cluster of Excellence MATISSE. We acknowledge the use of cleanroom facilities from the consortium "Salles Blanches Paris Centre - SBPC."

${ }^{1}$ E. Lhuillier, M. Scarafagio, P. Hease, B. Nadal, H. Aubin, X. Z. Xu, N. Lequeux, G. Patriarche, S. Ithurria, and B. Dubertret, Nano Lett. 16, 1282 (2016).

${ }^{2}$ J. Joo, J. S. Son, S. G. Kwon, J. H. Yu, and T. Hyeon, J. Am. Chem. Soc. 128, 5632 (2006).

${ }^{3}$ E. Lhuillier, S. Pedetti, S. Ithurria, B. Nadal, H. Heuclin, and B. Dubertret, Acc. Chem. Res. 48, 22 (2015).

${ }^{4}$ M. Nasilowski, B. Mahler, E. Lhuillier, S. Ithurria, and B. Dubertret, Chem. Rev. 116, 10934 (2016).

${ }^{5}$ L. C. Flatten, S. Christodoulou, R. K. Patel, A. Buccheri, D. M. Coles, B. P. L. Reid, R. A. Taylor, I. Moreels, and J. M. Smith, Nano Lett. 16, 7137 (2016).

${ }^{6}$ A. Riedinger, F. D. Ott, A. Mule, S. Mazzotti, P. N. Knüsel, S. J. P. Kress, F. Prins, S. C. Erwin, and D. J. Norris, "An intrinsic growth instability in isotropic materials leads to quasi-two-dimensional nanoplatelets," Nat. Mater. (published online).

${ }^{7}$ F. Fan, P. Kanjanaboos, M. Saravanapavanantham, E. Beauregard, G. Ingram, E. Yassitepe, M. M. Adachi, O. Voznyy, A. K. Johnston, G. Walters, G.-H. Kim, Z.-H. Lu, and E. H. Sargent, Nano Lett. 15, 4611 (2015).

${ }^{8}$ Z. Chen, B. Nadal, B. Mahler, H. Aubin, and B. Dubertret, Adv. Funct. Mater. 24, 295 (2014).

${ }^{9}$ A. W. Achtstein, A. Schliwa, A. Prudnikau, M. Hardzei, M. V. Artemyev, C. Thomsen, and U. Woggon, Nano Lett. 12, 3151 (2012).

${ }^{10}$ R. Benchamekh, N. A. Gippius, J. Even, M. O. Nestoklon, J.-M. Jancu, S. Ithurria, B. Dubertret, A. L. Efros, and P. Voisin, Phys. Rev. B 89, 35307 (2014).

${ }^{11}$ J. Q. Grim, S. Christodoulou, F. Di Stasio, R. Krahne, R. Cingolani, L. Manna, and I. Moreels, Nat. Nanotechnol. 9, 891 (2014).

${ }^{12}$ B. Guzelturk, Y. Kelestemur, M. Olutas, S. Delikanli, and H. V. Demir, ACS Nano 8, 6599 (2014). 
${ }^{13}$ C. She, I. Fedin, D. S. Dolzhnikov, A. Demortière, R. D. Schaller, M. Pelton, and D. V. Talapin, Nano Lett. 14, 2772 (2014).

${ }^{14}$ S. Ithurria and D. V. Talapin, J. Am. Chem. Soc. 134, 18585 (2012).

${ }^{15}$ B. Mahler, B. Nadal, C. Bouet, G. Patriarche, and B. Dubertret, J. Am. Chem. Soc. 134, 18591 (2012).

${ }^{16}$ A. Prudnikau, A. Chuvilin, and M. Artemyev, J. Am. Chem. Soc. 135, 14476 (2013).

${ }^{17}$ S. Pedetti, S. Ithurria, H. Heuclin, G. Patriarche, and B. Dubertret, J. Am. Chem. Soc. 136, 16430 (2014).

${ }^{18}$ B. Mahler, P. Spinicelli, S. Buil, X. Quelin, J.-P. Hermier, and B. Dubertret, Nat. Mater. 7, 659 (2008).

${ }^{19} \mathrm{P}$. Harrison, Quantum Wells, Wires and Dots, 2nd ed. (Wiley, Hoboken, NJ, 2005).

${ }^{20}$ O. Madelung, M. Schulz, and H. Weiss, Numerical Data and Functional Relationships in Science and Technology, New Series. Group III: Crystal and Solid State Physics. Semiconductors, Supply and Extension, Vol. III/ 17. Intrinsic Properties of Group IV Elements and III-V, II-VI, and I-VII Compounds (Springer, Berlin, 1982).

${ }^{21}$ S. Z. Karazhanov and L. C. L. Y. Voon, Semiconductors 39, 161 (2005).
${ }^{22}$ R. Dalven, Phys. Status Solidi B 48, K23 (1971).

${ }^{23}$ L. Quiroga, F. J. Rodríguez, A. Camacho, and C. Tejedor, Phys. Rev. B 42, 11198 (1990).

${ }^{24}$ S.-H. Wei and A. Zunger, Appl. Phys. Lett. 72, 2011 (1998).

${ }^{25}$ F. Polack, M. Silly, C. Chauvet, B. Lagarde, N. Bergeard, M. Izquierdo, O. Chubar, D. Krizmancic, M. Ribbens, J.-P. Duval, C. Basset, S. Kubsky, and F. Sirotti, AIP Conf. Proc. 1234, 185 (2010).

${ }^{26}$ D. D. Sarma, P. K. Santra, S. Mukherjee, and A. Nag, Chem. Mater. 25, 1222 (2013).

${ }^{27}$ B. Mahler, L. Guillemot, L. Bossard-Giannesini, S. Ithurria, D. Pierucci, A. Ouerghi, G. Patriarche, R. Benbalagh, E. Lacaze, F. Rochet, and E. Lhuillier, J. Phys. Chem. C 120, 12351 (2016).

${ }^{28}$ J. E. B. Katari, V. L. Colvin, and A. P. Alivisatos, J. Phys. Chem. 98, 4109 (1994).

${ }^{29}$ E. Lhuillier, S. Ithurria, A. Descamps-Mandine, T. Douillard, R. Castaing, X. Z. Xu, P.-L. Taberna, P. Simon, H. Aubin, and B. Dubertret, J. Phys. Chem. C 119, 21795 (2015).

${ }^{30}$ C. Wang, M. Shim, and P. Guyot-Sionnest, Science 291, 2390 (2001).

${ }^{31}$ H.-L. Yip and A. K.-Y. Jen, Energy Environ. Sci. 5, 5994 (2012). 